S. Ohtsuka $\cdot$ K. Murao $\cdot$ H. Imachi $\cdot$ W. M. Cao

X. Yu • J. Li • H. Iwama $\cdot$ N. C. W. Wong •

C. Bancroft • T. Ishida

\title{
Prolactin regulatory element binding protein as a potential transcriptional factor for the insulin gene in response to glucose stimulation
}

Received: 9 November 2005 / Accepted: 13 February 2006 / Published online: 20 April 2006

C) Springer-Verlag 2006

\begin{abstract}
Aims/hypothesis: Prolactin regulatory element binding (PREB) protein has been identified as a factor that regulates prolactin promoter activity in rat anterior pituitary. PREB is located not only in the anterior pituitary but also in pancreas; however its role in the pancreas is not known. We therefore examined the role of PREB in insulin gene expression. Materials and methods: To analyse the effects of PREB on insulin gene transcription, we employed the luciferase reporter gene assay and electrophoretic mobility shift assay (EMSA). In cells expressing or knocked down for PREB, insulin expression and secretion were determined. Results: PREB was located mainly in nuclei of rat pancreatic beta cells and its cell line, INS-1. A nuclear extract of INS-1 cells contained material that was recognised by PREB antiserum. This nuclear extract also showed insulin promoter binding activity that was super-shifted by PREB antiserum in EMSA studies. In the INS-1 cells, co-expression of PREB and the insulin promoter induced activity of the latter. The
\end{abstract}

S. Ohtsuka $\cdot$ K. Murao $(\bowtie) \cdot H$. Imachi

W. M. Cao $\cdot$ X. Yu $\cdot$ J. Li $\cdot$ T. Ishida

Division of Endocrinology and Metabolism,

Department of Internal Medicine,

Faculty of Medicine, Kagawa University,

1750-1, Miki-cho, Kita-gun,

Kagawa, Japan

e-mail: mkoji@kms.ac.jp

Tel.: +81-878-912145

Fax: $+81-878-912147$

H. Iwama

Information Technology Center, Kagawa University,

Kagawa, Japan

N. C. W. Wong

Departments of Medicine

and Biochemistry \& Molecular Biology, University of Calgary,

Calgary, AB, Canada

C. Bancroft

Department of Physiology and Biophysics,

Mount Sinai School of Medicine,

New York, NY, USA addition of glucose to the cells increased PREB expression. Deletional analysis of the insulin promoter showed that $\mathrm{A} 3$, a glucose-responsive cis-element in the insulin promoter, mediated the transcriptional effect of PREB. In addition, synthesised PREB bound the A3 element by EMSA, while a mutant of this motif in the insulin promoter abrogated the effect of PREB. Cells expressing or knocked down for PREB exhibited increased or decreased insulin expression, respectively. Conclusions/interpretation: These results demonstrate that PREB may contribute to the regulation of insulin gene transcription and insulin secretion in response to glucose stimulation.

Keywords A3 element - Beta cells - Glucose - Insulin · PREB - Prolactin - Promoter - Transcription

Abbreviations EMSA: electrophoretic mobility shift assay - LUC: luciferase - PCBE: PREB core binding element - PDX1: pancreatic and duodenal homeobox protein 1 PIT1: pituitary-specific transcription factor PKA: protein kinase A - PREB: prolactin regulatory element binding

\section{Introduction}

In pituitary lactotropic cells, prolactin is produced in a celltype-specific fashion. This selective expression arises from the actions of a pituitary-specific transcription factor (PIT1) that regulates expression of the gene encoding prolactin in these cells $[1,2]$. The PIT1 binding motif of the gene encoding prolactin was used to screen a rat pituitary cell line library [3] leading to the isolation of a cDNA that encoded prolactin regulatory element binding (PREB) protein. PREB binds to and activates the prolactin promoter but it can also mediate the actions of protein kinase $\mathrm{A}$ on the promoter [3]. The predicted amino acid sequence of PREB protein revealed the presence of three regions of high similarity to the WD-repeat consensus, and two potential trans-regulatory PQ-rich regions. Thus PREB 
belongs to the eukaryotic family of WD-repeat proteins. The highly conserved WD-repeats within PREB exhibit sequence similarity to a subset of this family comprised of proteins that act as gene regulators. PREB transcripts were detected not only in the pituitary, but also in heart, skeletal muscle and pancreas, with a high level of expression in the latter tissue [3]. However, the role of PREB in pancreas is unknown.

The insulin gene is expressed exclusively in pancreatic islet beta cells. This cell-specific expression is controlled by $c i s$-acting elements residing within -340 to -91 of the insulin gene and by the interaction of these motifs with cellular factors [4]. Site directed mutagenesis of the promoter showed that the A3 (-201 to -196$), \mathrm{C} 1$ (-115 to -107$)$, and E1 ( -100 to -91$)$ elements are essential for efficient expression of the insulin gene in beta cells [5-8]. Under physiological conditions, insulin gene transcription is controlled by the glucose concentration through a mechanism that regulates the activity of both $\mathrm{C} 1$ and $\mathrm{A} 3$ cis-acting elements [4]. The A3 element contributes to both beta-cell-selective $[6,7,9]$ and glucose-regulated transcription $[9,10]$ of the insulin gene.

The beta-cell-enriched homeodomain factor or pancreatic and duodenal homeobox protein 1 (PDX1) controls A3-element-directed transcription [7, 9, 11]. Previous studies showed that PDX1 bound to and trans-activated the insulin gene and it may be involved in the glucoseregulated transcription of the insulin gene, but other factors may also contribute to the actions of this site [9]. In this report, we examined the transcriptional effect of PREB on the pancreas-specific gene, insulin. We found that PREB bound to the insulin promoter at the site that also bound PDX1 and regulated insulin gene activity in response to glucose. These results suggest that PREB may play a role in glucose control of insulin gene transcription.

\section{Materials and methods}

\section{Cell culture}

The INS-1 cells originated from a rat insulinoma cell line (gift from C. B. Wollheim, University Medical Center, Geneva, Switzerland). We isolated pancreatic islets from adult Wister rats as described previously [12]. The protocol employed in this experiment was reviewed and approved by the Kagawa University Institutional Animal Care and Use Committee. COS-7 cells (American Type Culture Collection, Manassas, VA, USA) and MIN6 cells (gift from M. Miyazaki, Osaka University) were grown in DMEM supplemented with $10 \%$ FBS.

\section{Northern blot analysis}

Total RNA was extracted from the cells. A full-length cDNA of rat PREB was labelled with $\left[{ }^{32} \mathrm{P}\right] \mathrm{dCTP}$ $(9.2 \mathrm{MBq})$ by the random priming method (Takara, Tokyo, Japan). Blots were also probed with rat $\beta$-actin gene to assess equal loading of samples [13]. After autoradiography at room temperature for $24 \mathrm{~h}$, hybridisa-

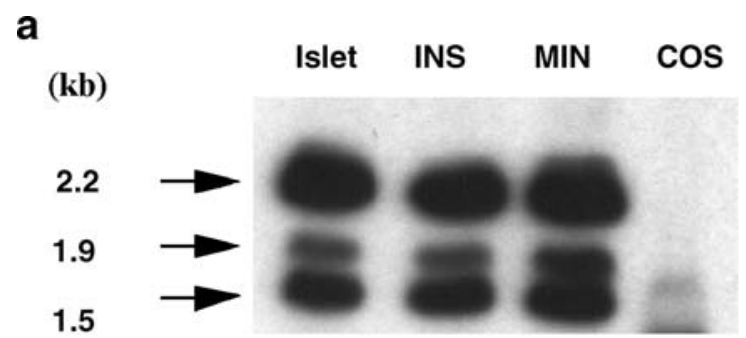

B-Actin

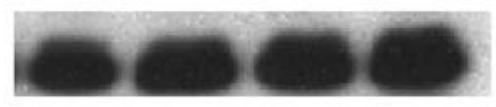

b
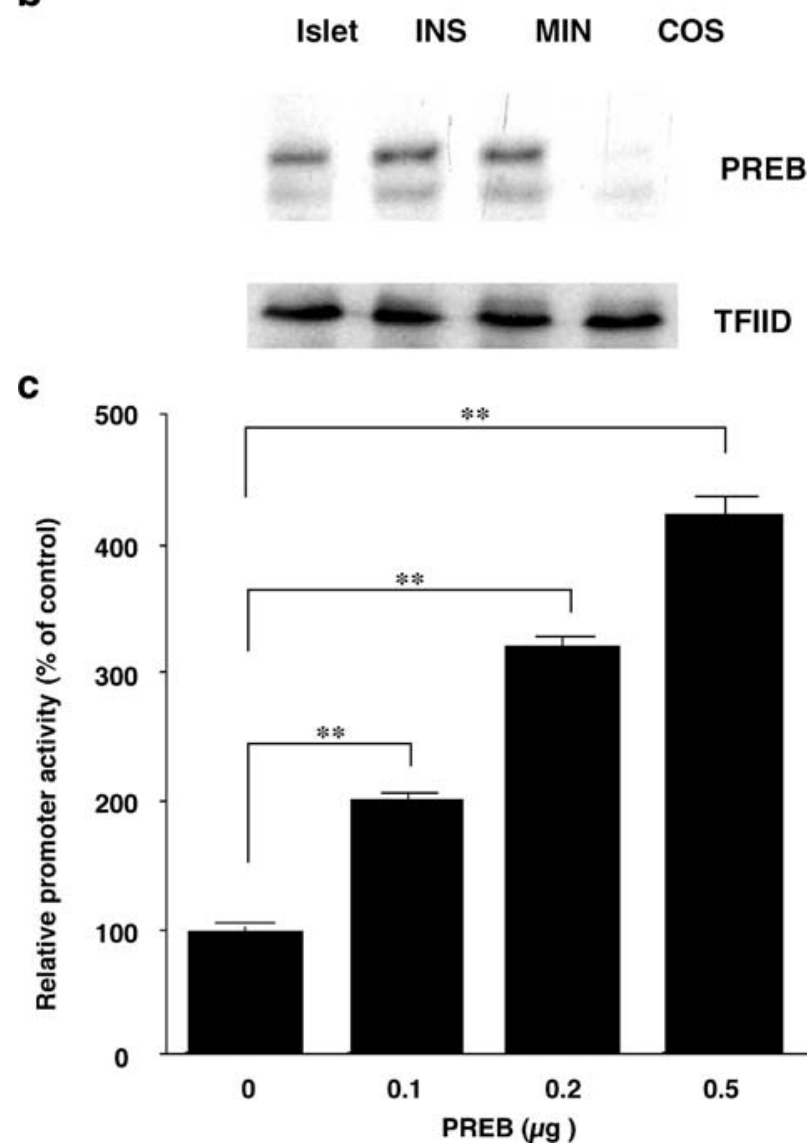

Fig. 1 Expression of PREB and effect of PREB on insulin promoter activity. a Presence of Preb mRNA in rat pancreatic islets (Islet), INS-1, MIN6 and COS-7 cells was examined by northern blot analysis of $2 \mu \mathrm{g}$ polyA RNA from these cells, with $\beta$-actin used as a control for equal RNA loading. b PREB protein expression. Nuclear extract from the cells was extracted and subjected to western blot analysis. Abundance of basal transcriptional factor (TFIID) served as a control. c Effect of PREB on insulin promoter activity: dishes $(60 \mathrm{~mm})$ of INS-1 cells were co-transfected with $1 \mu \mathrm{g}$ of $-238 \mathrm{WT}-$ LUC plus the indicated amounts of PREB expression vector. All assays were corrected for $\beta$-galactosidase activity and equal total amounts of protein per reaction were employed. The results are expressed as relative luciferase activity relative to that of control cells arbitrarily set at 100 . Each data point shows the mean and SE $(n=3)$ of separate transfections. ${ }^{* *} p<0.01$ 
tion signals were detected using a Bioimaging Analyzer (BAS 1000; Fuji Photo Film, Tokyo, Japan).

Electrophoretic mobility shift assay

Nuclear extracts from the cells that had been treated with various glucose concentrations for $24 \mathrm{~h}$ were prepared according to a technique described previously [14]. We used a synthetic DNA duplex spanning A3 (5'-CCTCT TAAGACTCTAATTACCCT-3'), mutant A3 probe (mt-A3) (p) (5'-CCTCTTAAGACTCGACTTACCCT-3'), mt-A3 (pm) (5'-CCTCTTAAGCACCTAATTACCCT-3'), mt-A3 (cm) (5'CCTAGGAAGACTCTAATTACCCT-3'), 1P (-66 TGCCT GATTATATATATATTCATGAAGGTGTCGAA-32) (Nihon Bioservice, Asagiri, Japan). Sequences were radiolabelled at the $5^{\prime}$-end by incubating each strand separately with $\left[\gamma\left[{ }^{32} \mathrm{P}\right]\right.$ ATP and polynucleotic kinase prior to annealing. All reactions were incubated at room temperature for $20 \mathrm{~min}$ and then separated on a native $6 \%$ polyacrylamide gel.

\section{Western blot analysis}

Cells were washed, scraped in PBS and lysed as described previously [15]. The membranes were incubated for $1 \mathrm{~h}$ at $4^{\circ} \mathrm{C}$ with $0.2 \%$ Tween 20 in PBS containing anti-PREB antiserum (diluted 1:250) as described previously [3]. Antibody binding was visualised using a chemiluminescence detection kit (ECL; Amersham Pharmacia Biotech, Buckinghamshire, UK). a

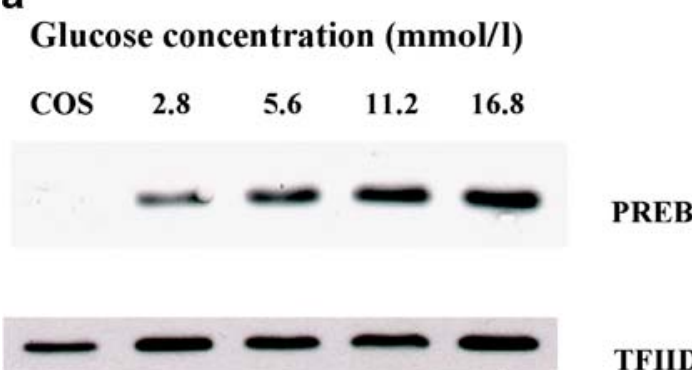

b

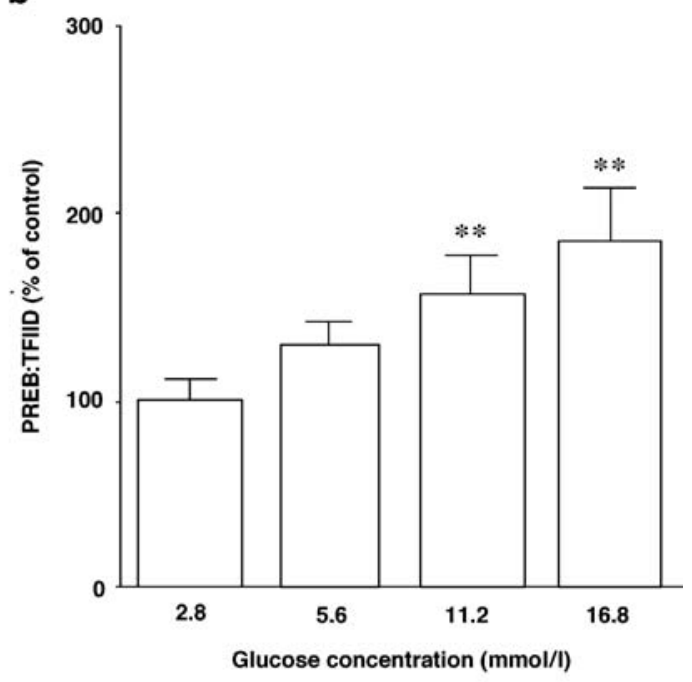

C

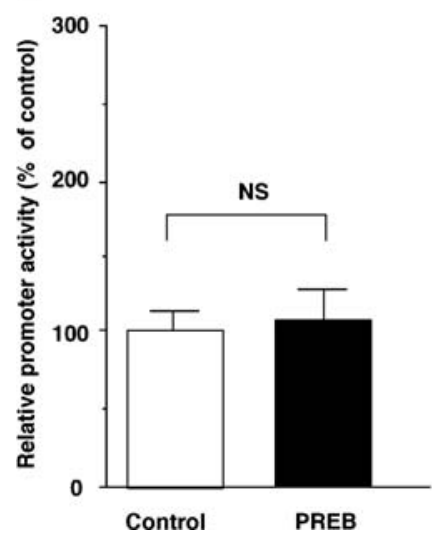

d

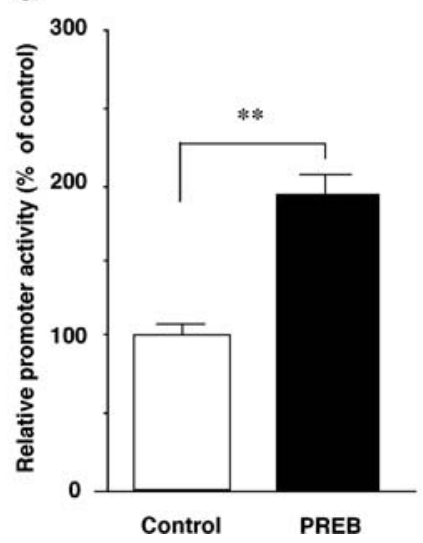

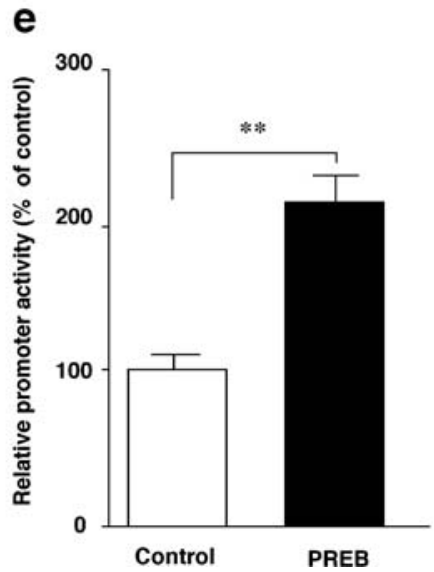

Fig. 2 Effects of glucose concentration on PREB protein accumulation in rat pancreatic islets. Nuclear extract of rat pancreatic islets was treated with varying concentrations of glucose for $24 \mathrm{~h}$. a PREB accumulation was examined by western blot. Basal transcriptional factor (TFIID) served as a control. COS, COS-7 cells as a negative control. b The ratio of PREB:TFIID, shown as percentage of control. The results are presented as the mean $\pm \mathrm{SE}$ of three experiments.
$* * P<0.01$. c-e Site-directed mutagenesis of the A3 abrogates the response to PREB. The reporter genes containing (c) -238 WT LUC, -238 A3 mutant LUC ( -238 A3m LUC), (d) -238 C1 mutant LUC $(-238 \mathrm{C} 1 \mathrm{~m}$ LUC), and (e) -238 E1 mutant LUC (-238 E1m LUC) were constructed, and cells transfected with the mutated reporter genes. Promoter activity was determined by luciferase assay. Data points show the mean and SE $(n=4)$ of separate transfections. ${ }^{* *} p<0.01$ 
In vitro transcription and translation

The pcDNA3.1 (+) vector carrying PREB cDNA or vector only was transcribed in vitro with T7 RNA polymerase (Gibco-BRL, Tokyo, Japan) according to the manufacturer's instructions. The RNA product was translated with a rabbit reticulocyte lysate system (Promega, San Luis Obispo, CA, USA).

Transfection of INS-1 cells and luciferase reporter gene assay

The reporter gene used in our studies was kindly provided by R. Stein (Vanderbilt University Medical Center, Nashville, TN, USA). Wild-type (-238 WT LUC) contains the gene encoding rat insulin II sequences spanning the region from -238 to +2 linked to the luciferase reporter gene [16]. The constructs, mutant types (-238 mA3-LUC, $-238 \mathrm{mC} 1-\mathrm{LUC},-238 \mathrm{mE} 1-\mathrm{LUC})$ lacking the consensus sequence $\mathrm{A} 3, \mathrm{C} 1$ and $\mathrm{E} 1$, respectively, were generated as described previously [17]. Site-directed mutagenesis of the A3 element within the first 238 bp of the rat II insulin gene promoter was performed according to the manufacture's instruction (Stratagene, La Jolla, CA, USA). In brief, the PREB core sequence of the consensus motif was disrupted by altering three base pairs $\left(5^{\prime}\right.$-ACT to $5^{\prime}$-CAC) in the A3 element derived from the parent construct -238 WT-LUC. Purified reporter plasmid was transfected into the cells using a conventional cationic liposome transfection method (Life Technologies, Gaithersburg, MD, USA). All assays were corrected for $\beta$-galactosidase activity; total amounts of protein per reaction were identical [14].

\section{Stable transfection}

The PREB expression vector was transfected into the cultured INS-1 cells by the conventional cationic liposome transfection method as described previously [18]. The transfected cells were selected by adding G418 to the media, and clones showing high PREB production were identified using western blot analysis. To determine cellular insulin content, INS-1 cells were incubated for $24 \mathrm{~h}$ with fresh culture medium and then rinsed in PBS and lysed in $0.1 \mathrm{~N} \mathrm{HCl}$ in $100 \%$ ethanol at $4^{\circ} \mathrm{C}$. The lysate was stored overnight at $-80^{\circ} \mathrm{C}$. The thawed lysate was vortexed and subjected to brief centrifugation at $10,000 \times g$. The resulting supernatant was diluted for measurement of insulin. The levels of immunoreactive insulin were quantified using a commercially available sandwich-type ELISA (Shibayagi, Tokyo, Japan).

\section{Transfection of siRNA}

The siRNAs were designed to target the following cDNA sequences: scrambled, 5'-CCGTTCTGTACAGGGAGT ACT-3'; and Preb siRNA, 5'-AATGGCGTGCACTTTCT a

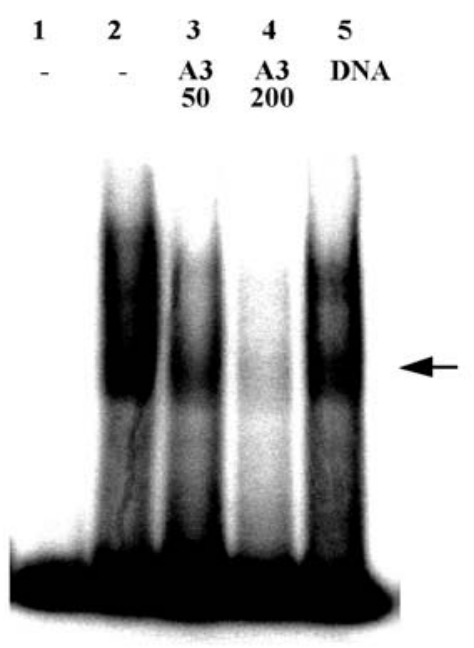

b
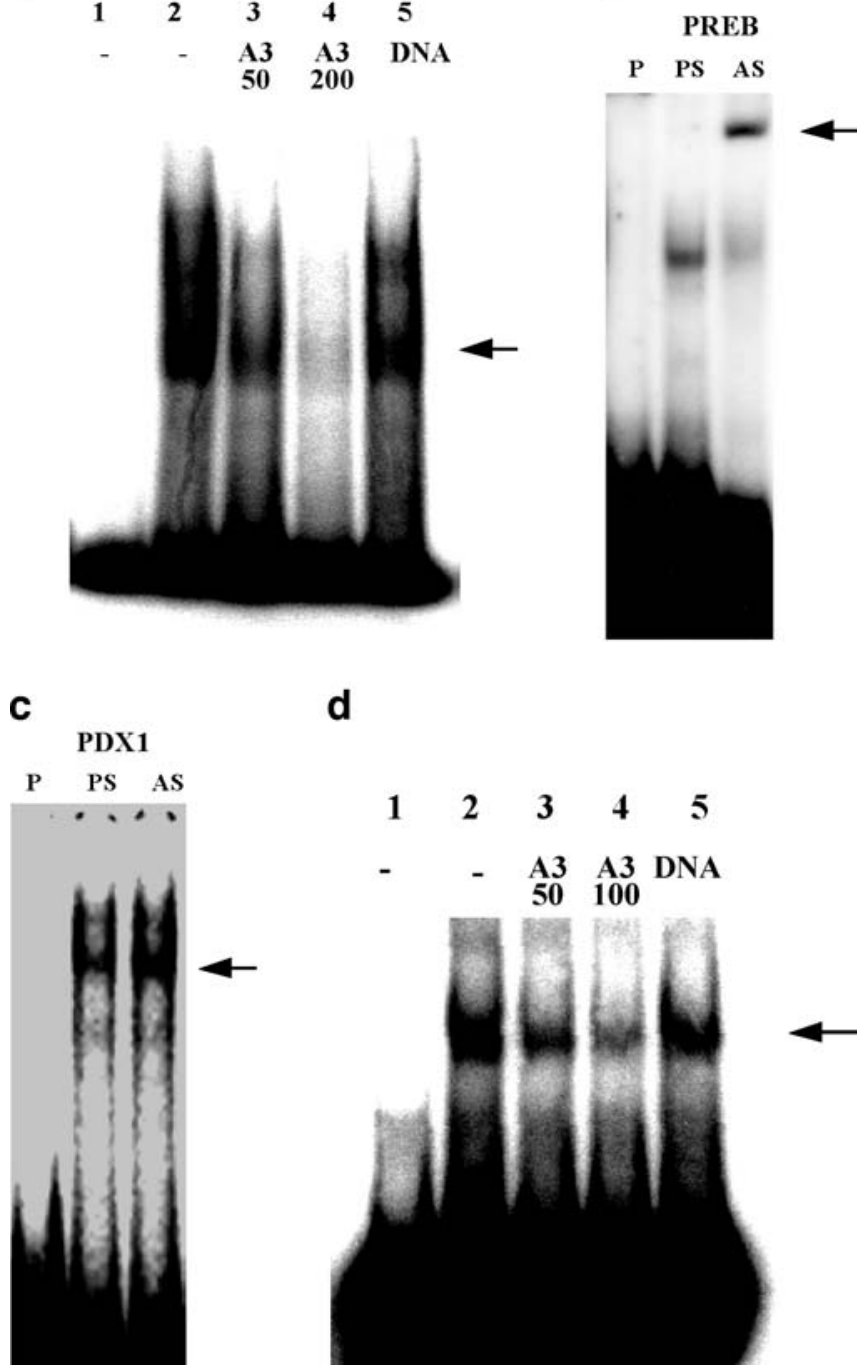

d

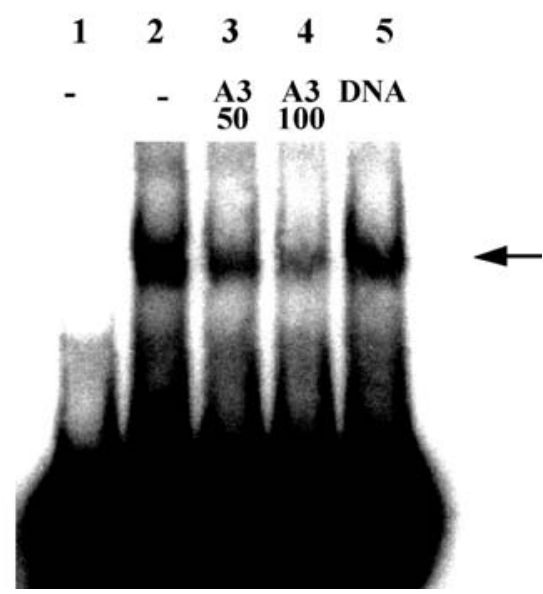

Fig. 3 PREB exhibits DNA sequence-specific binding to insulin promoter element A3. a Autoradiograph of EMSA of nuclear extract from INS-1 cells bound to radiolabelled 1P. Competition reactions showed binding pattern of nuclear protein with an indicated excess of cold A3 and nonspecific DNA. Lane 1, probe only; lane 2, no competitor; lane 3, 50-fold mol/1 excess of unlabelled A3; lane 4, 200 -fold $\mathrm{mol} / 1$ excess of unlabelled A3; lane 5, 200-fold mol/1 excess of unlabelled nonspecific DNA. Super shift assay with antiserum against PREB (b) or PDX1 (c). EMSA was carried out using the $1 \mathrm{P}$ oligonucleotide as probe. For lanes PS, nuclear extracts from INS-1 with $1 \mu$ l of pre-immune serum were added, whereas $1 \mu 1$ of antiserum raised against PREB (b) or PDX1 (c) was added for lanes AS. Arrows point to the 1P-specific complex. d Autoradiograph of EMSA reactions containing A3 probe and reticulocyte lysate that had been incubated without (lane I) and with (lanes 2-5) Preb mRNA. Competition reactions showed binding pattern of nuclear protein with an excess of cold A3 (lane 3, 50-fold mol/1 excess of unlabelled A3; lane 4, 100-fold mol/1 excess of unlabelled A3; lane 5, 50-fold mol/l excess of unlabelled nonspecific DNA)

GCAG-3' [3]. Transfection of Preb siRNA was performed using siPORT Amine (Ambion, Austin, TX, USA). Insulin mRNA expression was examined using northern blot analysis 3 days after transfection. 
a

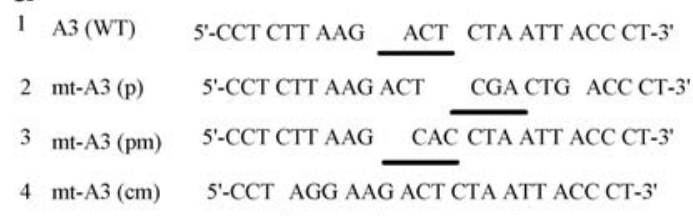

C

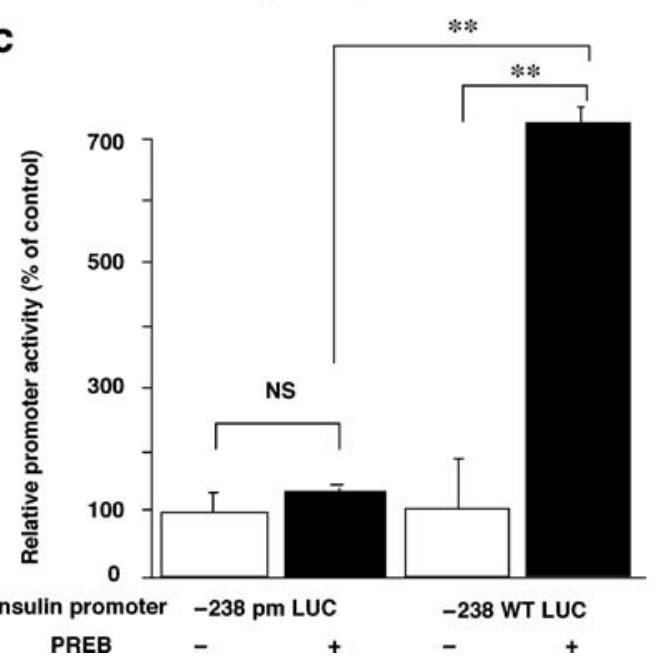

b P $1,2,3,4$,

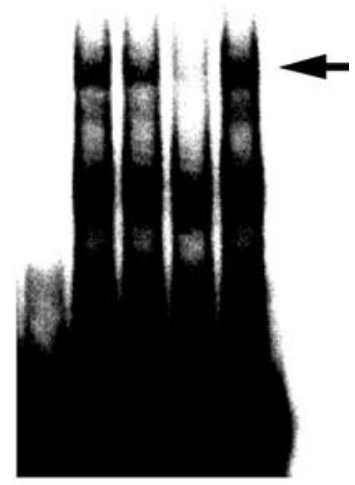

Fig. 4 The DNA target site in A3 cis-element for PREB. a Structure of A3 and sequences of mutated A3 oligonucleotides. Underlined sections: sites of the mutation. b Autoradiograph of EMSA reactions containing synthesised PREB protein by in vitro transcription/ translation and radiolabelled probe as indicated. Lane P, A3 (WT) probe only; lane 1, PREB plus A3 (WT) probe; lane 2, PREB plus mt-A3 (p) probe; lane 3, PREB plus mt-A3 (pm) probe; lane 4, PREB plus mt-A3 $(\mathrm{cm})$ probe. c Site-directed mutagenesis of the A3

\section{Statistical analysis}

Statistical comparisons were made by one-way analysis of variance and Student's $t$-test, with $p<0.05$ considered significant.

\section{Results}

Presence of the PREB transcript in the rat pancreatic islet and rat insulinoma cell line, INS-1

We examined PREB expression in rat pancreatic islets, the insulinoma cell lines, INS-1, MIN6, and COS-7. Results showed the presence of Preb mRNA of the predicted size [3] in rat pancreatic islet, INS-1 and MIN6, but as expected not in COS-7 cells (Fig. 1a). To further extend our previous finding that PREB is located in the nuclei of the cells [3], we isolated nuclear extract from rat pancreatic islets, INS-1, MIN6 and COS-7 cells. The results of these studies showed the presence of a major protein band of approximately $45 \mathrm{kDa}$ that matched the expected size of PREB protein (Fig. 1b), but there was no evidence of PREB in the cytosol of these cells (data not shown). abrogates the response to PREB. The PREB core sequence of consensus motif was disrupted by altering three base pairs ( $5^{\prime}$-ACT to $\left.5^{\prime}-\mathrm{CAC}\right)$ in the $\mathrm{A} 3$ element $(-238 \mathrm{pm}$ LUC) derived from the parent construct -238 WT-LUC as described in Materials and methods. INS-1 cells were co-transfected with $1 \mu \mathrm{g}$ of $-238 \mathrm{WT}-$ LUC or $-238 \mathrm{pm}$ LUC plus the PREB expression vector. Each data point shows the mean and SEM $(n=3)$ of separate transfections. $* * p<0.01$

Effects of PREB on the rat insulin promoter in INS-1 cells

To determine whether PREB affected transcriptional activity of the insulin promoter in INS-1 cells we used the -238 WT LUC construct comprised of the full-length rat insulin II promoter co-transfected into INS-1 cells with varying amounts of PREB expression vector. Results in Fig. 1c show that the presence of PREB induced up to a fourfold increase of insulin promoter activity, this induction being dependent on the amount of PREB. Next we examined the ability of PREB to regulate insulin gene transcription. To do this, we used COS-7 cells because western blot analysis of this cell line yielded undetectable endogenous levels of PREB (Fig. 1a). Exogenous PREB induced up to a threefold increase of insulin promoter activity in COS-7 cells (data not shown).

Glucose stimulates accumulation of PREB

protein in the nuclei of pancreatic islets

Since glucose is the most important regulator of insulin gene expression in pancreatic beta cells, we examined the effect of this metabolic regulator on PREB accumulation in rat pancreatic islets. Western blots probed with a PREB antiserum showed that the abundance of PREB protein 
a

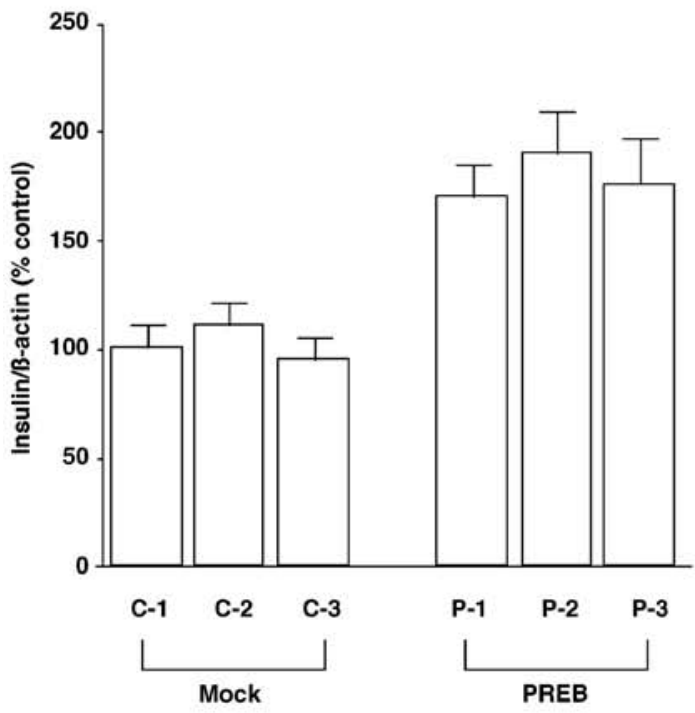

b

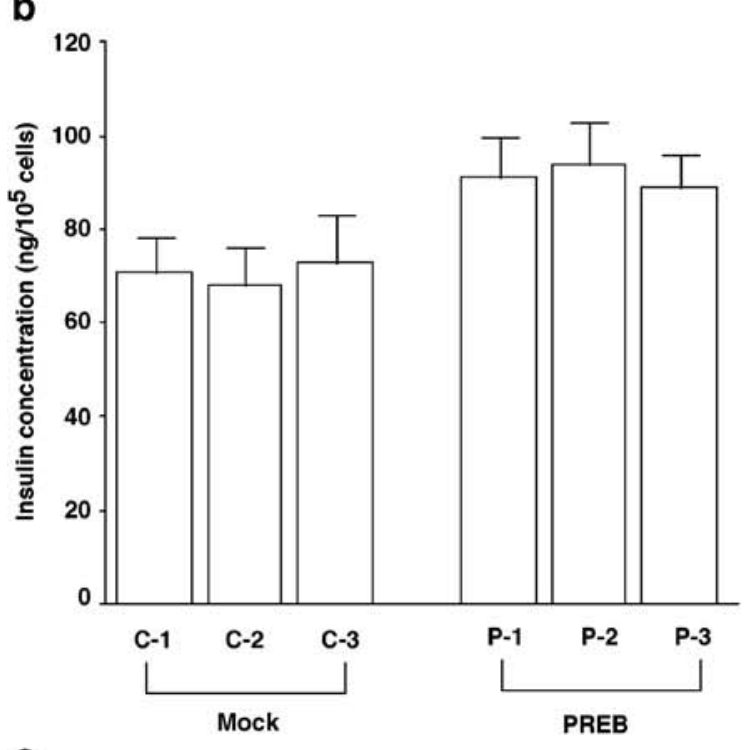

C

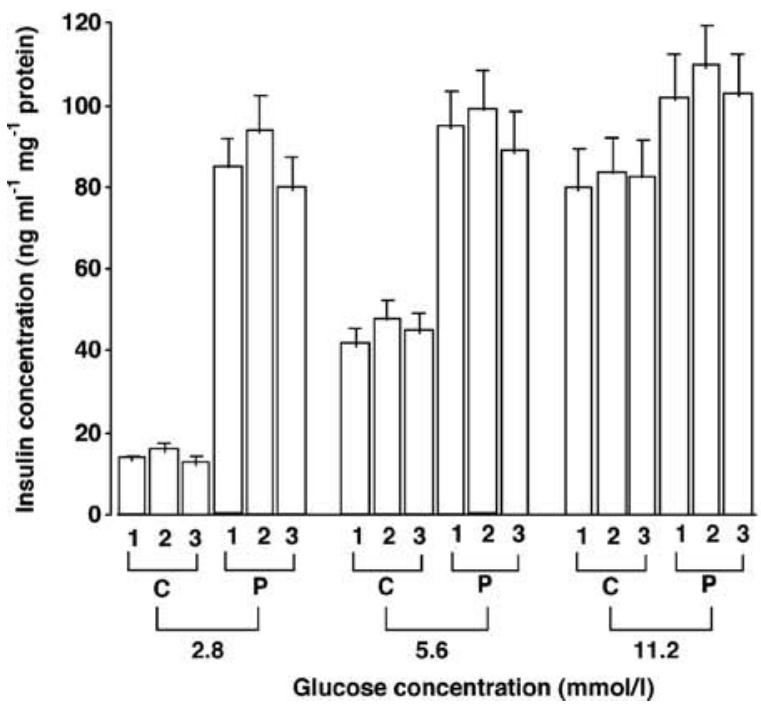

d

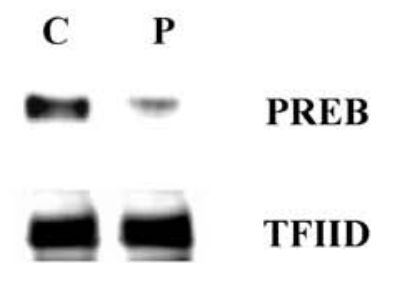

e
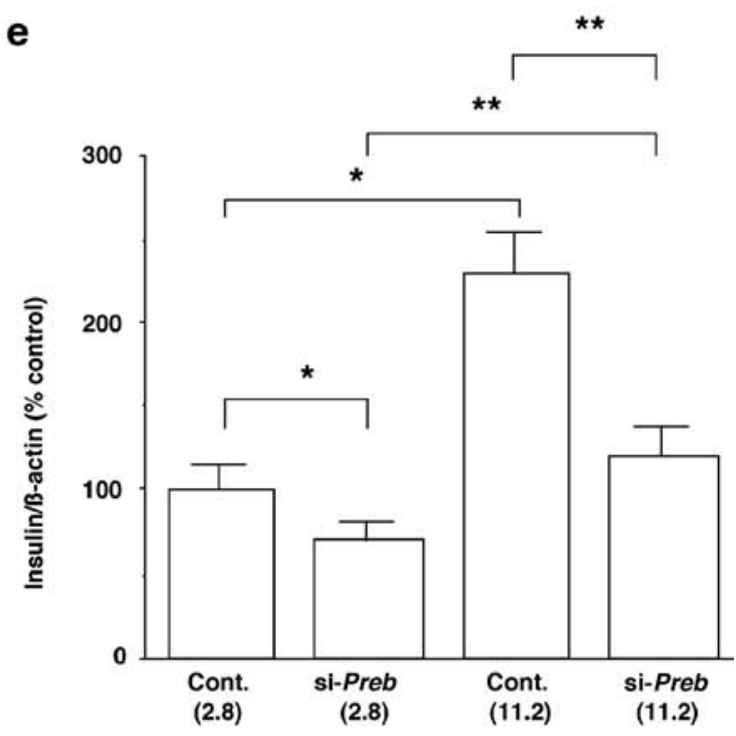
Fig. 5 Insulin gene expression in PREB-transfected cells. a Insulin mRNA of PREB-transfected INS-1 clones. The abundance of insulin/ $\beta$-actin mRNA levels was measured by northern blot analysis. The ratio of insulin mRNA to $\beta$-actin mRNA is shown as percentage of control. Values represent the mean of triplicate determinations. C-1, C-2, and C-3: empty vector, pcDNA3 transfected clones. P-1, P-2, and P-3: PREB-transfected clones. b Insulin content of PREB-transfected INS-1 clones. Insulin content was measured using ELISA (see Materials and methods). Values represent the mean of triplicate determinations. C-1, C-2, and C-3: empty vector, pcDNA3 transfected clones. P-1, P-2, and P-3: PREBtransfected clones. c Glucose-stimulated insulin secretion in transfected INS-1 clones. Insulin secretion in response to glucose as indicated was measured in the mock-transfected clones (C: C-1, C-2, C-3) and the PREB-transfected clones (P: P-1, P-2, P-3). Insulin secreted into the medium was measured with ELISA and normalised to total cellular protein. Values represent the mean of triplicate determinations. d Nuclear extracts of the cells treated with Preb siRNA were subjected to western blot to examine PREB expression. Basal transcription factor (TFIID) was used as a control. Lane $C$, scrambled siRNA; Lane P, Preb siRNA. An identical experiment independently performed gave similar results. e Effects of PREBknock down on insulin expression in INS-1 cells. Preb siRNA (si-Preb) or control siRNA (Cont) was transfected into INS-1 cells. Seventy-two hours after transfection, the abundance of insulin $/ \beta$ actin mRNA levels was measured using northern blot analysis. The ratio of insulin mRNA to $\beta$-actin mRNA is shown as percentage of control. Each data point shows the mean $\pm \operatorname{SE}(n=3)$ of separate experiments. Numbers in parentheses: glucose concentration ( $\mathrm{mmol} / \mathrm{l})$. ${ }^{*} p<0.01, * * p<0.005$ )

increased in response to glucose (Fig. 2) in a dosedependent manner. In contrast, the basal transcriptional factor TFIID was not affected by glucose concentration.

PREB interacts with the A3 cis-acting element of insulin promoter

To further examine whether the stimulating effect of glucose is mediated by PREB, three constructs containing a mutation of the glucose-response elements were created. The -238 A3m LUC (A3 deletion), $-238 \mathrm{C} 1 \mathrm{~m}$ LUC (C1 deletion), and $-238 \mathrm{E} 1 \mathrm{~m}$ LUC (E1 deletion) were derived from the parent construct -238 WT LUC by deleting these glucose-response motifs. The co-transfection of these reporter genes with PREB expression vector into INS-1 cells showed that PREB had no effect on the A3-deleted insulin promoter, but it still had an effect on C1 and E1 deletional mutants (Fig. 2c-e). These findings suggest that the A3 element is required for the actions of PREB on insulin promoter activity.

Next we examined whether the action of PREB affected the binding activity of nuclear proteins that interacted with the A3 cis-element. PREB binds directly to site $1 \mathrm{P}$ of the prolactin promoter [3]. We used electrophoretic mobility shift assay (EMSA) competition analysis of PREB binding to the $1 \mathrm{P}$ site in the presence of a 50 - or 200-fold molar excess of unlabelled A3 competitor DNA. Results (Fig. 3a) show that nuclear extract from INS-1 cells bound to $1 \mathrm{P}[3]$. The addition of excess A3 DNA competed for PREB binding to the radiolabelled 1P probe. Since several nuclear proteins are known to bind the A3 motif, we employed the super shift assay with a specific antiserum against PREB. Our results (Fig. 3b,c) show that the protein-DNA complex was shifted by the addition of anti-PREB antiserum but not by pre-immune serum or antiserum against PDX1 (Fig. 3c). To determine whether the binding activity in the INS-1 extract to the A3 motif was indeed due to PREB, we synthesised the protein using in vitro transcription/translation, and used EMSA to assess whether the in vitro synthesised PREB could bind radiolabelled A3 motif. We found that PREB expressed using in vitro transcription/translation bound the A3 element, and that excess unlabelled A3 DNA inhibited formation of the retarded complex (Fig. 3d). In brief, these results demonstrate that PREB can bind to the A3 motif of the insulin promoter and this cis-acting element may be involved in the regulation of the insulin promoter.

\section{PREB exhibits DNA sequence-specific binding to insulin promoter element $\mathrm{A} 3$}

To further define the cis-acting motif in the A3 cis-element that mediates the effect of PREB, we searched the promoter for known PREB response elements spanning the -213 to -192 fragment. Our search revealed one trinucleotide motif (ACT, Fig. 4a) corresponding to the deduced PREB core binding element (PCBE) of the prolactin gene [3]. To investigate whether the ACT motif is required for the effect of PREB on the insulin promoter, we synthesised several DNA fragments (mt-A3 [p], mt-A3 [pm], mt-A3 [cm]) for use as EMSA probes. Results (Fig. 4b) show that the mutation of this ACT sequence (mt-A3 [pm]) decreased PREB binding, but other mutations did not. Next we created a construct, $-238 \mathrm{pm} \mathrm{LUC}$, that contained a mutated putative PCBE (5'-ACT to $5^{\prime}$-CAC). Studies (Fig. 4c) showed that co-transfection of PREB does not stimulate the activity of the $-238 \mathrm{pm}$ LUC construct, but it does stimulate activity of the wild-type -238 WT LUC. These observations suggest that the putative PCBE in the A3 cis-element is required for the stimulatory action of PREB on the insulin promoter.

\section{Effect of PREB on insulin synthesis and secretion}

To examine the role of PREB on insulin synthesis, we established stable transfected INS-1 cells that expressed PREB. Several stably transfected clones containing PREB were isolated (data not shown). In the PREB-producing clones (P-1, P-2, P-3), insulin content (Fig. 5a) and mRNA (Fig. 5b) was significantly higher than in clones carrying pcDNA3 (C-1, C-2, C-3). Both mock and PREB-transfected cells were exposed to varying concentrations of glucose. In the PREB-transfected cells, insulin secretion was higher at all concentrations of glucose than in mocktransfected cells (Fig. 5c). As expected, the secretion of insulin following glucose stimulation also increased from mock-transfected cells. 
To further demonstrate whether increased insulin gene activity was affected by glucose-induction of PREB expression, we used siRNA to block PREB-expression. The expression of PREB was inhibited by Preb siRNAtreatment but not by a scrambled siRNA-treatment (Fig. 5d). INS-1 cells were exposed to PREB-specific or control siRNA and then treated with varying concentrations of glucose. As shown in Fig. 5e, insulin gene expression was increased in cells exposed to control siRNA following stimulation with $11.2 \mathrm{mmol} / 1$ glucose. In contrast, glucose induction of insulin expression was significantly suppressed in cells treated with Preb siRNA at low or high glucose concentrations. These findings may be consistent with the idea that glucose induction of insulin gene activity requires PREB.

\section{Discussion}

The PREB protein was recently isolated from a rat pituitary cDNA library, and shown to transactivate prolactin promoter activity [3]. Preb mRNA transcripts were present not only in pituitary, but a strong signal was also present in the pancreas. These findings led us to examine the potential role of PREB in pancreatic cell function. In this study, our results show PREB to be a transcription factor that was capable of enhancing insulin promoter activity and appeared to mediate insulin gene expression in response to glucose stimulation. A number of our observations suggested that PREB may play a role as a pancreatic cell transcription factor. First, antiserum raised against recombinant PREB detected a major protein that localised to the nucleus of pancreatic islets, MIN6, and INS-1 cells (Fig. 1). Secondly, Preb mRNA was expressed in pancreatic islets, MIN6, and INS-1 cells (Fig. 1). Thirdly, super-shift analysis of PREB motif binding activity confirmed its presence in the nuclear extract and localised the protein to the nucleus of the INS- 1 cells. Together these data strongly suggest that the protein recognised by the PREB antibody present in the nuclear extract of INS-1 cells is endogenous PREB, and that it is also a nuclear protein present in pancreatic beta cells.

The pituitary-specific transcription factor, PIT1, regulates expression of growth hormone, thyroid stimulating hormone, and also prolactin in the anterior pituitary [19]. PIT1 can stimulate activity of the prolactin promoter via binding to the $1 \mathrm{P}$ motif [20]. PREB protein is encoded by a $1.9-\mathrm{kb}$ transcript, which was shown to bind directly to site $1 \mathrm{P}$ of the prolactin promoter, rather than to a motif that is separate from that of the PIT1 site [4]. Analysis of the primary sequence of PREB revealed that it is a novel protein distinct from that of PIT1. PREB protein contained three motifs (WD I, WD II and WD III) possessing a significant degree of homology to the WD repeat consensus, thus suggesting that PREB was a member of the WD-repeat protein family [21]. The highly conserved WD-repeats within PREB exhibit sequence similarity to a subset of this family, consisting of proteins shown to act as gene regulators [21]. PREB effects transcriptional regula- tion in a manner quite different from other members of the WD-repeat protein family, because PREB can stimulate gene expression via direct binding to DNA [3]. Our results show that PREB can bind to the A3 DNA element of the insulin promoter and enhance insulin promoter activity not only in INS-1 insulinoma cells but also in non-pancreatic COS-7 cells.

The potential mechanism(s) of PREB action were examined by studying the promoter of the insulin gene. This promoter contains several cis-acting regulatory elements located within the 5 '-flanking region. Important transcriptional regulatory elements are present in the promoter regions of the insulin gene, two of the more important being the $\mathrm{A} 3$ and $\mathrm{C} 1$ elements [5-8]. $\mathrm{A} 3$ in the rat insulin promoter binds to PDX1, a homeodomain protein found in rat pancreatic islet cells. PDX1 transactivates the insulin gene in response to glucose $[11,22]$. Our findings show that PREB also binds specifically to the A3 element. EMSA analysis of PREB interaction with A3 or A3 mutants (Fig. 4b) implied that at least a portion of the PREB-binding site overlaps with that for PDX1 [11, 22]. As with PDX1, increasing concentrations of glucose also increased PREB activity. This observation suggests the possibility that PREB and PDX1 may co-occupy the A3 element, which would represent an opportunity for PREB and PDX1 to act in a complementary fashion on activity of the insulin promoter. The homeodomain protein PDX1 is known to interact with several transcription factors at the A3 element. For example, PDX1 regulation of insulin gene transcription involves interactions with the basic helixloop-helix protein E47/Pan1, BETA2/NeuroD1, and highmobility group protein I (Y) [23]. Although our results point to a possible cooperative role for PDX1 and PREB, further study will be necessary to determine whether this is physiologically significant in insulin gene transcription, possibly via interaction with other transcription vectors such as PDX1.

It is well known that glucose increases the concentration of both cAMP and $\mathrm{Ca}^{2+}$ in pancreatic beta cells [24, 25]. These changes trigger insulin secretion and synthesis in pancreatic beta cells [26]. The subsequent rise in intracellular concentrations of cAMP enhances glucose-mediated insulin secretion via protein kinase A (PKA)-dependent and independent pathways [27, 28]. We previously reported that PREB can mediate PKA stimulation of the prolactin promoter activity [3] suggesting a role for this protein in cAMP-mediated transcriptional responses. In this study, we showed that INS-1 cells stably producing PREB exhibit increased insulin secretion compared to mock-transfected cells. A possible model for this type of cellular role for PREB may involve the activation of PREB via PKA-mediated phosphorylation. Although it is not yet known whether PREB can serve as a PKA substrate either in vitro or in vivo, the predicted sequence of this protein does contain a number of motifs that resemble PKA phosphorylation consensus sites [29]. Further studies will be needed to determine the specific regulatory mechanisms that enable PREB to regulate insulin gene transcription. 
In summary, we have demonstrated that PREB can function as a transcriptional regulator of insulin promoter activity. PREB, like PDX1, binds to the glucose response element $\mathrm{A} 3$ of the insulin promoter, and cells expressing PREB increase insulin secretion. Further investigations will be needed to shed more light on a possible physiological role for PREB in pancreatic beta cells, both in glucose metabolism and in insulin gene expression.

Acknowledgements $\mathrm{K}$. Murao is supported in part by a Grantin-Aid for Scientific Research 14770601. We thank K. Yamaji and $\mathrm{K}$. Ueeda for their technical assistance.

\section{References}

1. Andersen B, Rosenfeld MG (1994) Pit-1 determines cell types during development of the anterior pituitary gland. A model for transcriptional regulation of cell phenotypes in mammalian organogenesis. J Biol Chem 269:29335-29338

2. Okimura Y, Howard PW, Maurer RA (1994) Pit-1 binding sites mediate transcriptional responses to cyclic adenosine 3 ',5'monophosphate through a mechanism that does not require inducible phosphorylation of Pit-1. Mol Endocrinol 8:1559-1565

3. Fliss MS, Hinkle PM, Bancroft C (1999) Expression cloning and characterization of PREB (prolactin regulatory element binding), a novel WD motif DNA-binding protein with a capacity to regulate prolactin promoter activity. Mol Endocrinol 13:644-657

4. Servitja JM, Ferrer J (2004) Transcriptional networks controlling pancreatic development and beta cell function. Diabetologia 47:597-613

5. Crowe DT, Tsai MJ (1989) Mutagenesis of the rat insulin II 5'flanking region defines sequences important for expression in HIT cells. Mol Cell Biol 9:1784-1789

6. German MS, Moss LG, Wang J, Rutter WJ (1992) The insulin and islet amyloid polypeptide genes contain similar cellspecific promoter elements that bind identical beta-cell nuclear complexes. Mol Cell Biol 12:1777-1788

7. Peshavaria M, Gamer L, Henderson E, Teitelman G, Wright CV, Stein R (1994) XIHbox 8, an endoderm-specific Xenopus homeodomain protein, is closely related to a mammalian insulin gene transcription factor. Mol Endocrinol 8:806-816

8. Whelan J, Poon D, Weil PA, Stein R (1989) Pancreatic betacell-type-specific expression of the rat insulin II gene is controlled by positive and negative cellular transcriptional elements. Mol Cell Biol 9:3253-3259

9. Petersen HV, Serup P, Leonard J, Michelsen BK, Madsen OD (1994) Transcriptional regulation of the human insulin gene is dependent on the homeodomain protein STF1/IPF1 acting through the CT boxes. Proc Natl Acad Sci USA 91:10465-10469

10. Marshak S, Totary H, Cerasi E, Melloul D (1996) Purification of the beta-cell glucose-sensitive factor that transactivates the insulin gene differentially in normal and transformed islet cells. Proc Natl Acad Sci USA 93:15057-15062

11. Ohlsson H, Karlsson K, Edlund T (1993) IPF1, a homeodomain-containing transactivator of the insulin gene. EMBO J $12: 4251-4259$
12. Lacy PE, Kostianovsky M (1967) Method for the isolation of intact islets of Langerhans from the rat pancreas. Diabetes $16: 34-39$

13. Murao K, Imachi H, Sayo Y et al (1999) A product of growth arrest-specific gene 6 modulates scavenger receptor expression in human vascular smooth muscle cells. FEBS Lett 459:363-366

14. Murao K, Wada Y, Nakamura T, Taylor AH, Mooradian AD, Wong NC (1998) Effects of glucose and insulin on rat apolipoprotein A-I gene expression. J Biol Chem 273:18959-18965

15. Murao K, Terpstra V, Green SR, Kondratenko N, Steinberg D, Quehenberger O (1997) Characterization of CLA-1, a human homologue of rodent scavenger receptor $\mathrm{BI}$, as a receptor for high density lipoprotein and apoptotic thymocytes. J Biol Chem 272:17551-17557

16. Yu X, Murao K, Sayo Y et al (2004) The role of calcium/ calmodulin-dependent protein kinase cascade in glucose upregulation of insulin gene expression. Diabetes 53:1475-1481

17. Sharma A, Stein R (1994) Glucose-induced transcription of the insulin gene is mediated by factors required for beta-cell-typespecific expression. Mol Cell Biol 14:871-879

18. Cao WM, Murao K, Imachi H et al (2004) A mutant highdensity lipoprotein receptor inhibits proliferation of human breast cancer cells. Cancer Res 64:1515-1521

19. Scully KM, Jacobson EM, Jepsen K et al (2000) Allosteric effects of Pit-1 DNA sites on long-term repression in cell type specification. Science 290:1127-1131

20. Ingraham HA, Flynn SE, Voss JW et al (1990) The POU-specific domain of Pit-1 is essential for sequence-specific, high affinity DNA binding and DNA-dependent Pit-1-Pit-1 interactions. Cell 61:1021-1033

21. Neer EJ, Schmidt CJ, Nambudripad R, Smith TF (1994) The ancient regulatory-protein family of WD-repeat proteins. Nature 371:297-300

22. Ohlsson H, Thor S, Edlund T (1991) Novel insulin promoterand enhancer-binding proteins that discriminate between pancreatic alpha- and beta-cells. Mol Endocrinol 5:897-904

23. McKinnon CM, Docherty K (2001) Pancreatic duodenal homeobox-1, PDX-1, a major regulator of beta cell identity and function. Diabetologia 44:1203-1214

24. Charles MA, Lawecki J, Pictet R, Grodsky GM (1975) Insulin secretion. Interrelationships of glucose, cyclic adenosine 3:5-monophosphate, and calcium. J Biol Chem 250:6134-6140

25. Wollheim CB, Sharp GW (1981) Regulation of insulin release by calcium. Physiol Rev 61:914-973

26. Nielsen DA, Welsh M, Casadaban MJ, Steiner DF (1985) Control of insulin gene expression in pancreatic beta-cells and in an insulin-producing cell line, RIN-5F cells. I. Effects of glucose and cyclic AMP on the transcription of insulin mRNA. J Biol Chem 260:13585-13589

27. Gonzalez GA, Montminy MR (1989) Cyclic AMP stimulates somatostatin gene transcription by phosphorylation of CREB at serine 133. Cell 59:675-680

28. Kwok RP, Lundblad JR, Chrivia JC et al (1994) Nuclear protein CBP is a coactivator for the transcription factor CREB. Nature 370:223-226

29. Kennelly PJ, Krebs EG (1991) Consensus sequences as substrate specificity determinants for protein kinases and protein phosphatases. J Biol Chem 266:15555-15558 